\title{
Anomalous density of states of a Luttinger liquid in contact with a superconductor
}

\author{
C. Winkelholz ${ }^{(1)}$, Rosario Fazio ${ }^{(2)}$, F.W.J. Hekking ${ }^{(3)}$, and Gerd Schön ${ }^{(1,4)}$ \\ (1) Institut für Theoretische Festkörperphysik, Universtität Karlsruhe, 76128 Karlsruhe, Germany \\ (2) Istituto di Fisica, Università di Catania, viale A. Doria 6, 95129 Catania, Italy \\ ${ }^{(3)}$ Cavendish Laboratory, University of Cambridge, Madingley Road, Cambridge CBз OHE, UK \\ (4) Department of Technical Physics, Helsinki University of Technology, 02150 Espoo, Finland
}

\begin{abstract}
We study the frequency and space dependence of the local tunneling density of states of a Luttinger liquid (LL) which is connected to a superconductor. This coupling strongly modifies the single-particle properties of the LL. It significantly enhances the density of states near the Fermi level, whereas this quantity vanishes as a power law for an isolated LL. The enhancement is due to the interplay between electron-electron interactions and multiple back-scattering processes of low-energy electrons at the interface between the LL and the superconductor. This anomalous behavior extends over large distances from the interface and may be detected by coupling normal probes to the system.
\end{abstract}


Transport in low-dimensional structures is strongly influenced by electron-electron interactions. A paradigm model to describe interactions in one-dimensional systems is the Luttinger liquid (LL). The low-lying excitations of the electron system consist of charge and spin waves, rather than quasiparticles $[1,2]$. As a consequence, the presence of a barrier in the liquid leads to perfectly reflecting (for repulsive interactions) or transmitting (for attractive interactions) behavior at low energies [3].

One of the most striking characteristic properties of Luttinger liquids is the behavior of the density of states (DOS) close to the Fermi energy. Contrary to Fermi liquids, whose quasiparticle residue is finite, LLs have a DOS which vanishes near the Fermi energy as a power law,

$$
N(\omega) \sim \omega^{\left(g_{\rho}+4 / g_{\rho}-4\right) / 8}
$$

The exponent $g_{\rho}$ depends on the strength of the electron-electron interaction: it is smaller (larger) than two for repulsive (attractive) interactions. In the non-interacting case $g_{\rho}=2$, and the DOS is constant as in Fermi liquids [4].

Recently it became possible to fabricate interfaces between a superconductor $(\mathrm{S})$ and a two-dimensional electron gas (2DEG) [5]. An excess low-voltage conductance due to Andreev scattering has been observed in Nb-InGaAs junctions [6], as well as a supercurrent through a 2DEG in an InGaAs/In AlGaAs heterostructure with Nb contacts [7,8]. If the 2DEG is gated to form a quantum wire, it should be possible to study transport through Superconductor - Luttinger Liquid (S-LL) interfaces. The Josephson current through a S-LL-S system has been calculated $[9,10]$, as well as the $I-V$ characteristics of a tunnel junction between a superconductor and a chiral LL [11]. The latter can be realized, e.g., in the fractional quantum Hall regime.

The proximity effect [12] modifies the properties of a normal metal $(\mathrm{N})$ in contact with a superconductor. The leakage of Cooper pairs induces a non-vanishing pair amplitude in $\mathrm{N}$, defined as $F(\vec{r})=\left\langle\psi_{\uparrow}(\vec{r}) \psi_{\downarrow}(\vec{r})\right\rangle$, where $\psi_{s}(\vec{r})$ is the annihilation operator for an electron with spin $s$. The pair amplitude is a two-particle property, related to the probability to find two time-reversed electrons at a position $\vec{r}$. In a clean normal metal at zero temperature, $F(\vec{r})$ decays as $1 / r$ away from the N-S interface. In a LL with repulsive interaction in contact with a superconductor, $F(\vec{r})$ decays as $1 / r^{\gamma}$, where $\gamma>1$ depends on the strength of interaction [10]. These results hold as long as $\vec{r}$ lies within the temperature-dependent coherence length $\xi_{N}=\hbar v_{F} / k_{B} T$ from the interface to the superconductor. At larger distances, $F(\vec{r})$ decays exponentially on the length scale $\xi_{N}$. The reason for the decay of the pair amplitude is that the two electrons loose their relative phase coherence over this distance. Single electrons, however, loose phase coherence only at a much larger distance, namely the phase-breaking length $L_{\phi}$. Indeed, recent experiments $[8,13]$ have shown that interference effects due to single quasiparticles in N-S systems persist over distances much larger than $\xi_{N}$. We, therefore, expect quite generally a considerable influence of superconductivity on single particle properties over distances where the pair amplitude has already decayed.

In order to investigate these properties in a strongly interacting system we study the local single-particle density of states (DOS) of a LL in contact with a superconductor. We find that the local DOS is substantially enhanced near the Fermi energy as compared to the power law decay of an isolated LL (cf. Eq.(1)). This result should be contrasted with the behavior of the pair amplitude (a two-particle property) which is suppressed in the interacting system. 
The anomalous enhancement is a result of the interplay between the scattering of low-energy electrons at the S-LL interface and the electron-electron interactions in the LL [14]. As for the space dependence, the DOS does not decay in the same fashion as the pair amplitude away from the S-LL interface. It remains enhanced up to distances of the order of the mean free path, which may be much larger than $\xi_{N}$ for a clean quantum wire. Hence, the effect cannot simply be explained in terms of a finite density of Cooper pairs in the LL. Both the frequency and the space dependence of the DOS can be detected experimentally by coupling normal metal tunneling probes to the LL at some distance from the superconductor.

In the inset of Fig. 1 we schematically draw a 'clean' S-LL interface, consisting of a LL in good contact with $\mathrm{S}$. The shaded area indicates a tunnel junction between the LL and a normal metal probe. We will also study a LL of finite length connected to two superconductors (S-LL-S system). In such a system Andreev bound states exist below the gap [15]. Finally, we will comment on the case of a LL connected to $S$ by means of a tunnel barrier. The anomalous enhancement of the DOS is found in this case as well.

The Hamiltonian of a LL can be written in bosonized form as $(\hbar=1)$

$$
\hat{H}_{L}=\frac{1}{2} \sum_{j} v_{j} \int d x\left[\frac{g_{j}}{2}\left(\nabla \phi_{j}\right)^{2}+\frac{2}{g_{j}}\left(\nabla \theta_{j}\right)^{2}\right],
$$

where $j=\rho, \sigma$, and $v_{j}=\left(2 / g_{j}\right) v_{F}$ are the renormalized interaction-dependent Fermi velocities. We restrict ourselves to repulsive, spin-independent interactions; hence $g_{\rho}<2$ and $g_{\sigma}=2$. The Fermi field operators are decomposed in terms of right- and left-moving Fermion operators $\psi_{+, s}$ and $\psi_{-, s}$, respectively, $\psi_{s}=e^{i k_{F} x} \psi_{+, s}+e^{-i k_{F} x} \psi_{-, s}$, where $k_{F}$ is the Fermi wave vector. The fields $\psi_{ \pm, s}$ in turn can be expressed through Boson operators

$$
\psi_{ \pm, s}^{\dagger}=\sqrt{\rho_{0}} e^{i \sqrt{\pi}\left[ \pm \phi_{s}(x)+\theta_{s}(x)\right]},
$$

where $\theta_{s}=\frac{1}{\sqrt{2}}\left(\theta_{\rho}+s \theta_{\sigma}\right)$ and $\phi_{s}=\frac{1}{\sqrt{2}}\left(\phi_{\rho}+s \phi_{\sigma}\right)$. The density of electrons per spin in the LL is $\rho_{0}=k_{F} / 2 \pi$. Maslov et al. [10] recently developed a bosonization scheme to treat clean S-LL interfaces. For a LL coupled to two superconductors at a distance $L$, they obtained the following normal mode expansion for the the fields

$$
\begin{aligned}
& \theta_{\rho}(x)=\sqrt{\frac{\pi}{2}}(J+\chi) \frac{x}{2 L}+\frac{i}{2} \sqrt{\frac{g_{\rho}}{2}} \sum_{q>0} \gamma_{q} \sin (q x)\left(\hat{b}_{\rho, q}^{\dagger}-\hat{b}_{\rho, q}\right) ; \\
& \theta_{\sigma}(x)=\frac{1}{\sqrt{\pi}} \theta_{\sigma}^{(0)}+\frac{i}{2} \sqrt{\frac{g_{\sigma}}{2}} \sum_{q>0} \gamma_{q} \sin (q x)\left(\hat{b}_{\sigma, q}^{\dagger}+\hat{b}_{\sigma, q}\right) ; \\
& \phi_{\sigma}(x)=\sqrt{\frac{\pi}{2}} M \frac{x}{2 L}+\frac{i}{2} \sqrt{\frac{2}{g_{\sigma}}} \sum_{q>0} \gamma_{q} \sin (q x)\left(\hat{b}_{\sigma, q}^{\dagger}-\hat{b}_{\sigma, q}\right) ; \\
& \phi_{\rho}(x)=\frac{1}{\sqrt{\pi}} \phi_{\rho}^{(0)}+\frac{i}{2} \sqrt{\frac{2}{g_{\rho}}} \sum_{q>0} \gamma_{q} \sin (q x)\left(\hat{b}_{\rho, q}^{\dagger}+\hat{b}_{\rho, q}\right) .
\end{aligned}
$$

Here, $\hat{b}_{j, q}^{(\dagger)}$ are Bose operators and $\gamma_{q}=\exp \{-q \alpha / 2 \pi\}$ where $\alpha$ is a short range cut-off. The phase difference between the two superconductors is $\chi ; J$ and $M$ describe the topological excitations satisfying the constraint $J+M=$ odd. Finally, $\theta_{\sigma}^{(0)}$ and $\phi_{\rho}^{(0)}$ are canonically conjugate to $J, M$ respectively. The local density of states (per spin) of the LL measured at 
a distance $x$ from the superconducting contact is obtained from the retarded one-electron Green's function of the LL, $G_{R}\left(x, x^{\prime} ; t\right) \equiv-i\left\langle\left\{\psi_{s}(x, t), \psi_{s}^{\dagger}\left(x^{\prime}, 0\right)\right\}\right\rangle \theta(t)$,

$$
N(x, \omega)=-\frac{1}{\pi} \mathcal{I} \mathrm{m} \int_{-\infty}^{\infty} d t e^{i \omega t} G_{R}(x, x ; t) \quad .
$$

We first discuss the space and frequency dependence of the DOS of a LL contacted at $x=0$ with a superconductor, which corresponds to the limit $L \rightarrow \infty$ in the mode expansion given by Eqs. (4) - (7). In this case only the non-zero modes $(q>0)$ contribute to the local DOS. The correlation function $\left\langle\psi_{s}^{\dagger}(x, t) \psi_{s}(x, 0)\right\rangle$ can be evaluated using the boson representation Eq. (3) with the result

$$
\begin{aligned}
&\left\langle\psi_{s}^{\dagger}(x, 0) \psi_{s}(x, t)\right\rangle=2 \rho_{0} \prod_{j=\rho, \sigma}\left(\frac{\alpha^{2}+(2 x)^{2}}{\alpha^{2}}\right)^{\gamma_{j}}\left(\frac{\alpha^{2}}{\left(\alpha-i v_{j} t\right)^{2}}\right)^{\eta_{j}} \\
& \times\left[\frac{\alpha^{2}}{\left(\alpha-i\left(2 x+v_{j} t\right)\right)\left(\alpha+i\left(2 x-v_{j} t\right)\right)}\right]^{\gamma_{j}},
\end{aligned}
$$

at a distance $x$ from the LL-S interface, where $\gamma_{j}=\left(g_{j} / 16-1 /\left(4 g_{j}\right)\right)$ and $\eta_{j}=\left(g_{j} / 16+\right.$ $\left.1 /\left(4 g_{j}\right)\right)$. At small energies the DOS behaves as

$$
N_{S-L L}(\omega) \sim \omega^{g_{\rho} / 4-1 / 2}
$$

The exponent of the DOS is negative $\left(g_{\rho}<2\right)$, which implies a strong enhancement at low energies whereas in the absence of $\mathrm{S}$ the DOS of the LL vanishes at the Fermi energy. The presence of the superconductor thus changes the properties of the Luttinger liquid in a qualitative way. Recently, Oreg and Finkel'stein [14] have found a similar enhancement of the local DOS of a LL in the presence of an impurity. They interpret their result as a consequence of the interplay between the back-scattering induced by the impurity and the repulsive interactions in the LL. A similar interplay exists in our system. Although we consider a clean S-LL interface, backscattering is induced by the superconducting gap, which reflects low-energy electrons either directly or via (multiple) Andreev processes. The enhanced DOS as a function of frequency, Eq. (10), is schematically drawn in Fig. 1; for comparison we also show the vanishing DOS in absence of the superconductor, Eq. (1).

At low energies $\omega$ the enhancement of the DOS persists over large distances $x(\omega) \sim v_{\rho} / \omega$ from the interface. On the other hand, the induced pair amplitude in the LL, which is characteristic of the presence of the superconductor, decays as a power [10] of the distance $x$. This profound difference in the space dependence demonstrates that the DOS provides different information compared to the proximity effect. The reason why the DOS does not approach the well-known behaviour of an Luttinger liquid far from the superconducting contact is in part related to the fact that we are considering a clean wire. In this case the states in the LL are extended and the DOS enhancement does not depend on $x$.

We now turn to the properties of the DOS for a S-LL-S system. The two superconductors are separated by the distance $L$ and are kept at a phase difference $\chi$. The latter can be achieved, e.g., by embedding this junction in a SQUID. As we consider a LL of finite length, the topological excitations should be taken into account; moreover the contribution from the non-zero modes consists of a discrete sum rather than a continuous integral over $q$-states. The correlator reads 


$$
\left\langle\psi_{ \pm, s}^{\dagger}(x, 0) \psi_{ \pm, s}(x, t)\right\rangle=e^{i \pi v_{F}(1 \pm \chi / \pi) t / 2 L} \prod_{j=\rho, \sigma} D_{j}(x, t)
$$

The exponential prefactor originates from the topological part; we used the fact that $J=1$ and $M=0$ in the ground state for $-\pi<\chi<\pi$. The $\chi$-dependence is related to the phasedependent shift of the Andreev levels (see below). The contribution from the non-zero modes is given by

$$
\begin{aligned}
D_{j}(x, t)= & \rho_{0}\left(\frac{\left(1-e^{-\pi(\alpha+2 i x) / L}\right)\left(1-e^{-\pi(\alpha-2 i x) / L}\right)}{\left(1-e^{-\pi \alpha / L}\right)^{2}}\right)^{\gamma_{j}}\left(\frac{1-e^{-\pi\left(\alpha-i v_{j} t\right) / L}}{1-e^{-\pi \alpha / L}}\right)^{-2 \eta_{j}} \\
& \times\left(\frac{\left(1-e^{-\pi \alpha / L}\right)^{2}}{\left(1-e^{\pi\left[\alpha-i\left(2 x+v_{j} t\right)\right]}\right)\left(1-e^{\pi\left[\alpha+i\left(2 x-v_{j} t\right)\right]}\right)}\right)^{\gamma_{j}} .
\end{aligned}
$$

As in the previous case of a single S-LL interface, the anomalous behavior of the DOS at low $\omega$ extends over large distances, (measured now relative to the position of one of the interfaces), hence the phase-dependent contribution to the DOS persists over distances much larger than the Josephson coupling. If the interaction constant can be written as a ratio of two integers $\left(g_{\rho}=m_{0} / n_{0}\right)$, we can express the DOS, using Eq. (11), as

$$
N(x, \omega)=\rho_{0} \sum_{s, \pm} \sum_{n} a_{n}(x)\left[\delta\left(\omega-E_{ \pm, n}\right)+\delta\left(\omega+E_{ \pm, n}\right)\right] .
$$

Here $a_{n}(x)$ are the Fourier coefficients of the function $D(x, t)$ corresponding to the energies

$$
E_{ \pm, n}=E_{F}\left(2 \frac{n}{n_{0}}+1\right) \frac{\pi}{2 L k_{F}} \pm v_{F} \frac{\chi}{2 L}
$$

where $E_{F}$ is the Fermi energy. The $E_{ \pm, n}$ are the energies of the Andreev levels [15] in the interacting quantum wire. The phase-difference $\chi$ lifts the degeneracy for right- and left moving electrons, giving rise to the Josephson effect. In the noninteracting case $\left(g_{\rho}=2\right)$, all the $\delta$-functions have the same weight and the local DOS shows a peak whenever the frequency $\omega$ coincides with an Andreev level $E_{ \pm, n}$. When the electron-electron interaction is switched on, the charge and spin part in Eq. (12) obtain different periodicities due to spincharge separation. As a consequence the coefficients $a_{n}$ show a more structured behaviour. In Fig. 2 the DOS is plotted for $g_{\rho}=1$ as a function of the frequency and of the distance from one of the two superconductors. For clarity we use a realistic, broadened version of the $\delta$ functions in Eq. (13). We, further, fixed the phase difference $\chi$ between the superconductors to zero. One clearly sees a strong enhancement of the DOS close to the interface. Away from the superconductor the DOS remains enhanced, but the energy scale of the enhancement is reduced to lower frequencies. The oscillatory contribution to the DOS is reminiscent of the Friedel-oscillations, characterized by a period $2 k_{F}$. In the general case $\chi \neq 0$, the DOS for the right moving electrons differs from that of the left moving electrons due to the phase factor in Eq. (11). Although this leads to a more complicated dependence of DOS on $x$ and $\omega$, the anomalous enhancement is still present.

So far we discussed the case in which the S-LL interface has a high transparency. Let us shortly comment on the opposite limit, in which the Luttinger liquid is connected to the superconductor by a tunnel junction. In this case at low energies, we find for the DOS close to the junction $N_{S-L L} \sim \omega^{\left(g_{\rho} / 2-1\right)+\left(1 / 2 g_{\rho}-g_{\rho} / 8\right)}$. Although the exponent is different from the 
one appearing in Eq. (10), the DOS is clearly enhanced. Moreover, also in this case the enhancement is found regardless of the distance from the junction.

In summary we considered the DOS of a Luttinger liquid in contact with a superconductor. We studied specifically the cases of a single S-LL interface and a S-LL-S system. Contrary to the well-known behavior in Luttinger liquids, the presence of the superconducting contact strongly enhances the local DOS close to the Fermi energy, and this behavior extends to large (energy-dependent) distances from the interface. Our results can be verified experimentally [16], e.g., by means of the setup drawn in the inset of Fig. 1. We imagine connecting the LL by means of a tunnel junction to a normal metal (at a distance $x$ from the interface) and measuring the $I-V$ characteristic of this junction. If there were no superconductor the conductance of the normal metal-LL junction would go to zero as the temperature (voltage, frequency) is lowered. The presence of the superconductor leads to an excess conductance at the junction.

\section{ACKNOWLEDGMENTS}

We thank C. Bruder for numerous important suggestions and W. Belzig and M. Fabrizio for useful discussions. The support of the Deutsche Forschungsgemeinschaft, through

SFB 195, the European Community through contract ERB-CHBI-CT94-1764, and the A.v.Humboldt award of the Finish Academy of Sciences (GS) is gratefully acknowledged. 


\section{REFERENCES}

[1] V.J. Emery, in Highly Conducting One-Dimensional Solids, edited by J.T. Devreese, R.P. Evrard, and V.E. van Doren (Plenum, New York, 1979); J. Sólyom, Adv. Phys. 28, 201 (1979).

[2] Spin charge separation has been observed in quantum wires, see A.R. Goñi, A. Pinczuk, J.S. Weiner, J.M. Calleja, B.S. Dennis, L.N. Pfeiffer, and K.W. West, Phys. Rev. Lett. 67, 3298 (1991).

[3] C.L. Kane and M.P.A. Fisher, Phys. Rev. Lett. 68, 1220 (1992); K.A. Matveev and L.I. Glazman, Phys. Rev. Lett. 70, 990 (1993); M. Fabrizio, A.O. Gogolin and S. Scheidl, Phys. Rev. Lett. 72, 2235 (1994).

[4] Measurements of the density of states indicate Luttinger liquid behaviour in Bechgaard salts, see B. Dardel, D. Malterre, M. Grioni, Y. Baer, J. Voit, and D. Jérôme, Europhys. Lett. 24, 687 (1993).

[5] See several articles in Mesoscopic Superconductivity, eds. F.W.J. Hekking, G. Schön, and D.V. Averin, Physica B 203 (1994).

[6] A. Kastalsky, A.W. Kleinsasser, L.H. Greene, R. Bhat, F.P. Milliken, and J.P. Harbison, Phys. Rev. Lett. 67, 3026 (1991).

[7] J. Nitta, T. Azaki, H. Takayanagi, and K. Arai, Phys. Rev. B 46, 14286 (1992).

[8] A. Dimoulas, J.P. Heida, B.J. van Wees, T.M. Klapwijk, W. v.d. Graaf,and G. Borghs, Phys. Rev. Lett. 74, 602 (1995).

[9] R. Fazio, F.W.J. Hekking, and A.A. Odintsov, Phys. Rev. Lett. 74, 1843 (1995).

[10] D.L. Maslov, M. Stone, P.M. Goldbart, and D. Loss, to be published in Phys. Rev. B.

[11] M.P.A. Fisher, Phys. Rev. B 49, 14550 (1994).

[12] G. Deutscher and P.G. de Gennes, in Superconductivity, edited by R.D. Parks (Marcel Dekker, NY 1965), vol. II, p. 1005.

[13] H. Courtois, Ph. Gandit, D. Mailly, and B. Pannetier, Phys. Rev. Lett. 76, 130 (1996).

[14] A similar anomalous enhancement of the local DOS has been recently found by Oreg and Finkel'stein for a LL with a backscattering defect. (Y. Oreg and A. Finkel'stein, cond/mat 9601020 (unpublished))

[15] I.O. Kulik, Zh. Eksp. Teor. Fiz. 57, 1745 (1969) [Sov. Phys. JETP 30, 944 (1970)].

[16] This setup has been proposed by D. Esteve et al. (private communication) to study the DOS of a diffusive conductor connected to a superconductor. A theoretical analysis has been performed by W. Belzig, C. Bruder, and G. Schön, to be published in Phys. Rev. B. 


\section{FIGURES}

FIG. 1. Schematic dependence of DOS on frequency for a pure LL (dashed line) and for a LL connected to S (solid line). Inset: Luttinger liquid, connected adiabatically to a superconductor. The shaded area indicates a tunnel junction with a normal metal used to measure the DOS in the LL at a distance $x$ from the interface.

FIG. 2. The local DOS for a S-LL-S system for particles of species $p= \pm$, plotted as a function of the frequency $\omega$ and the distance $x$ from one of the S-LL interfaces. We took $g_{\rho}=1$ (repulsive interactions); the $\delta$-functions of Eq. (13) have been smeared, using peaked Lorentzians with a width of the order the level spacing. Furthermore, $L k_{F}=10^{6}, N_{L}=\rho_{0} / E_{F}, \chi=0$. 


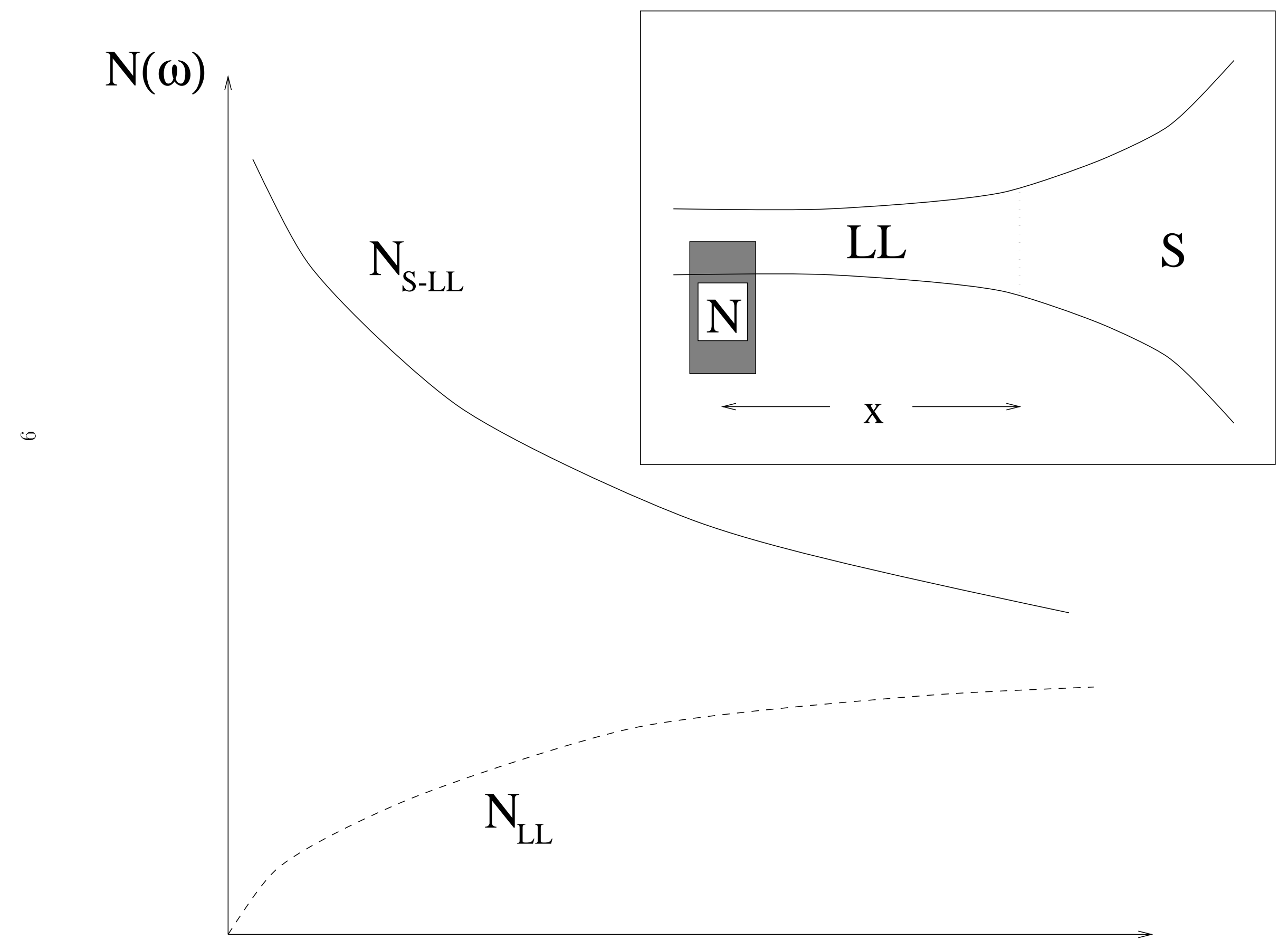




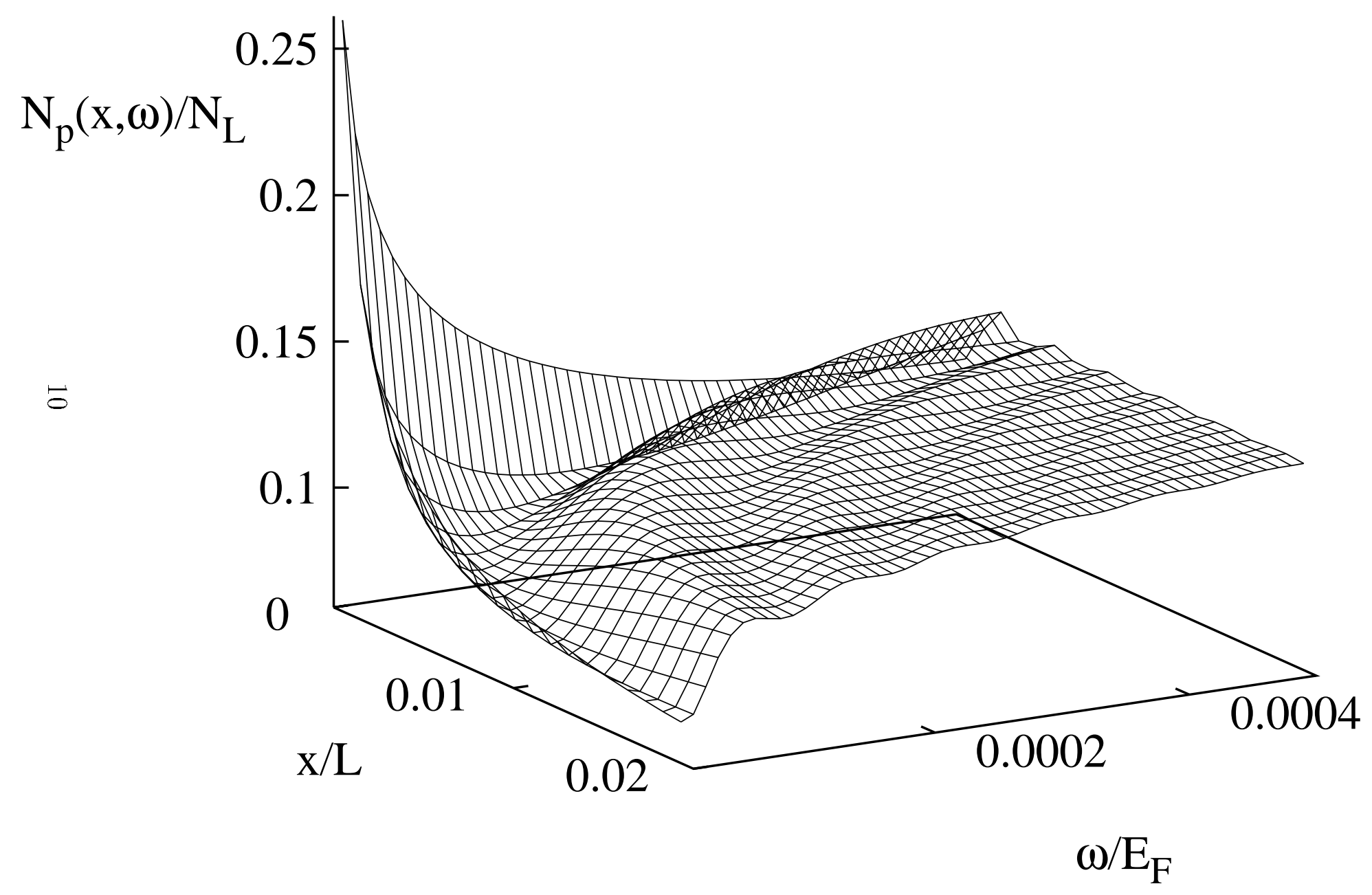

